\title{
DESIGN STUDY FOR SC PROTON LINAC ACCELERATING CAVITIES.
}

\author{
E. Zaplatine*, W. Braeutigam, S. Martin, \\ Forschungszentrum Juelich, Germany
}

\section{Abstract}

A 70-1334 MeV superconducting proton linac is under consideration as a possible version for the high energy part of European Spallation Source accelerator. In this paper we describe two alternative options of an accelerating structure $(500 \mathrm{MHz})$ for this machine. First is the 5-cell elliptical cavities designed for the $\beta=0.4-0.9$. The second type is a spoke cavity extended to multigap design $(\beta=0.3-0.5)$. Results of numerical simulations are compared with low level experimental data. A full scale SC elliptical cavity prototype is under construction in collaboration with ACCEL.

\section{INTRODUCTION}

In some recently launched projects[1]-[3] for high intensity proton beam acceleration the possibility to use superconducting cavities is under investigation. For this purpose a well established "elliptical" (Fig. 1) $\beta=1$ cavity shape is adapted for much slower proton beams with $\beta$ range from 0.4 to 0.9 . At the same time from mechanical calculations such type cavity use for $\beta$ lower than 0.5 is accomplished with a need of serious mechanical structure stiffeners. As a possible alternative a so called spoke cavity[4] is under consideration. During past year, at Forschungszentrum Juelich, we have been looking at the possibility to use SC cavities in European Spallation Source project (ESS)[5].

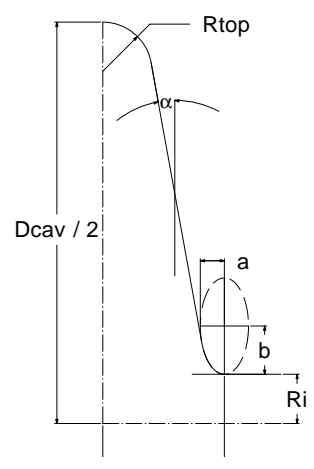

Figure 1: Elliptical Cavity Geometry (1/4 of cell is shown)

\section{ELLIPTICAL CAVITY}

Usually, an elliptical cavity design is a compromize between various geometric parameters which should define a most optimal cavity shape in terms of an accelerator purpose. Within a SC proton linac design there is a need of grouping of cavities with different $\beta=v / c$ values.

\footnotetext{
* On leave from the Joint Institute of Nuclear Research, Dubna
}

It means that the process of cavity designs for SC linac becomes time consuming. Here we present some basics which can help to obtain rather fast the main cavity parameters and which we used for ESS project. A further cavity optimization can be made afterwards but we believe that an improvement will be within $5 \%$.

The main advantage of any SC cavity is a possibility of high accelerating electric field maintaince $\left(E_{a c c}\right)$. There are two characteristics which limit in principle an acheivable value of $E_{a c c}$. They are the peak surface electric field $\left(E_{p k}\right)$ and the peak surface magnetic field $\left(H_{p k}\right) . H_{p k}$ is important because a superconductor will quench above the critical magnetic field. $E_{p k}$ is important because of the danger of field emission in high electric field regions. All these mean that to maximize the accelerating field first of all it is therefore important during a cavity design to minimize the ratios of peak fields to the accelerating field. There are some more figures of merit to compare different designs such as power dissipation $P_{c}$, a quality factor $\mathrm{Q}$ and shunt impedance $R_{s h}$. But these parameters are not so crucial to the cavity design and may be varyed in some limits without any sufficient harm for a system in whole. Here we should mention also such figure like the cavity apperture (bore radius in elliptical cavity design $R_{i}$ ). This characteristic is obtained in conjuction with beam dinamic calculations and is defined as a first. The choice of $R_{i}$ limits $E_{p k}$ (Fig. 2) and $H_{p k}$, defines cell-to-cell coupling in multicell cavity, influences the shunt impedance value and field flatness.

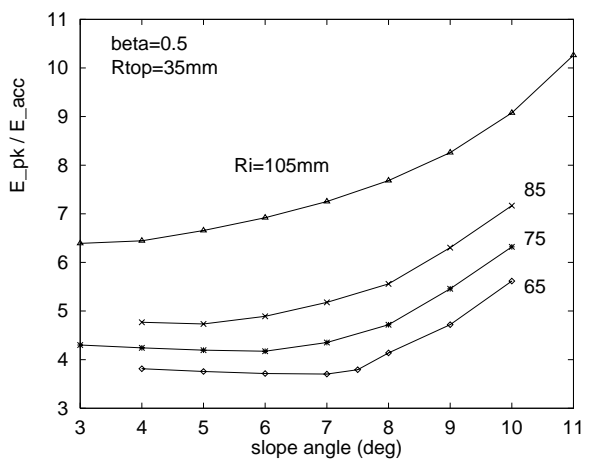

Figure 2: Maximum Electric Surface Field to Accelerating Field Ratio vs. Slope Angle $\alpha$

We start an elliptical cavity design with $R_{i}$ definition. Now, as to concern the cavity shape design itself there are some geometric characteristics (dome radius $R_{t o p}$, slope angle $\alpha$, ellipse axis) which should be defined for a most optimal cavity shape in terms of mentioned above RF parameters. And as a next step of design we made $E_{p k} / E_{a c c}$ and $H_{p k} / E_{a c c}$ investigations on slope angle $\alpha$ value. The 
reason of this is that an optimal value of $\alpha$ could be define unique if to consider dependences of $E_{p k} / E_{a c c}$ (a cavity frequency $500 \mathrm{MHz}$ ) (Fig. 3). From these grafs one may make a decision about a cavity shape. These calculations have been done by means of 2D cavity simulation code SUPERFISH[6]. The ellips parameters have been defined by program automatically to satisfy the fixed cell length $\beta \lambda / 2$. After this set of simulations the choice of $R_{i}$ should be checked. Fig. 4 shows dependences of the cavity coupling on iris radius for the chosen above optimal in terms of $\alpha$ points.

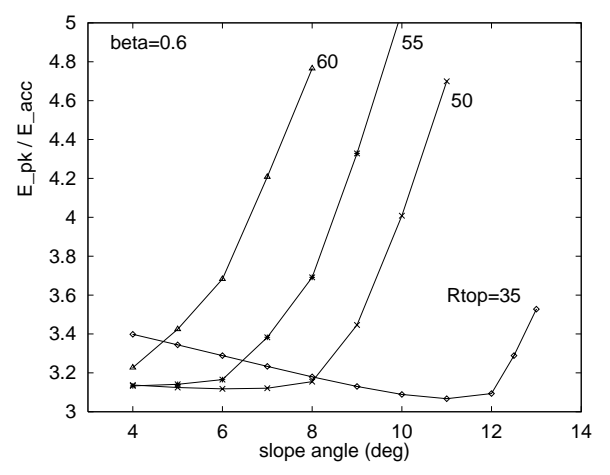

Figure 3: Maximum Electric Surface Field to Accelerating Field Ratio vs. Cavity Slope Angle $\alpha$

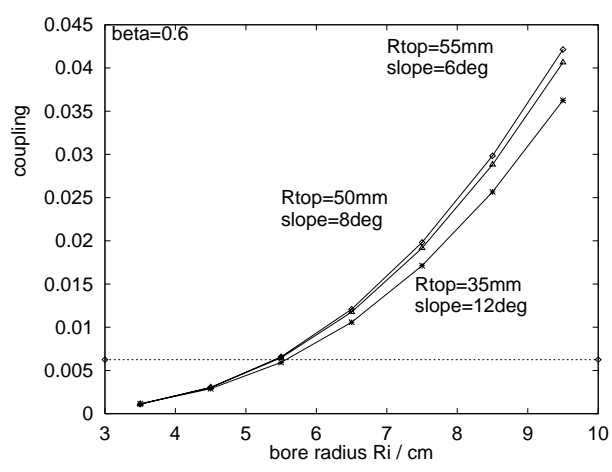

Figure 4: Cavity Coupling vs. Iris Radius

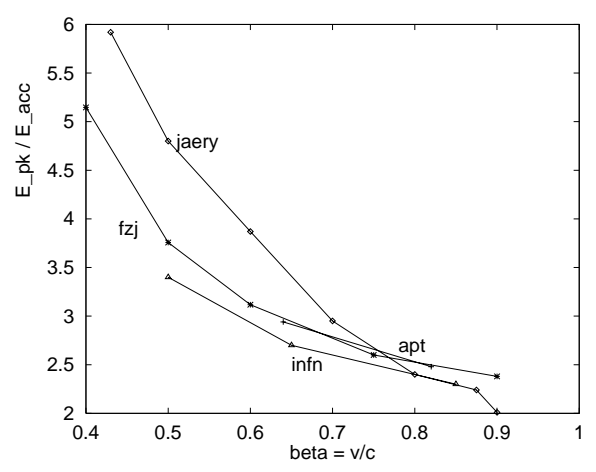

Figure 5: Maximum Electric Surface Field to Accelerating Field Ratio

The results of our calculations are presented on Fig. 5 along with results from other calculations[1]-[3]. These results reffer to the projects with quite different basic purposes and parameters (pulsed and cw, different final energies and RF frequencies), but all sets are for the same apperture $\left(R_{i}=65 \mathrm{~mm}\right.$ for FZJ).

Table 1 lists the main parameters of elliptical cavity designs for different $\beta$ values. Here we kept coupling approximately constant for all $\beta$ 's.

Table 1: Some Parameters to Compare Elliptical Cavities with Different $\beta=v / c$

\begin{tabular}{|l|c|c|c|c|c|}
\hline$\beta$ & $\mathbf{0 . 4}$ & $\mathbf{0 . 5}$ & $\mathbf{0 . 6}$ & $\mathbf{0 . 7 5}$ & $\mathbf{0 . 9}$ \\
\hline aperture $R_{i}(\mathrm{~cm})$ & 4.5 & 5.0 & 5.5 & 6.0 & 6.5 \\
dome $R_{\text {top }}(\mathrm{cm})$ & 2.5 & 3.5 & 4.5 & 6.5 & 8.5 \\
slope $\alpha(\mathrm{deg})$ & 6 & 7.5 & 10 & 12 & 14 \\
cell-length $(\mathrm{cm})$ & 12 & 0.15 & 0.18 & 0.225 & 0.27 \\
transit time factor & .775 & .772 & .768 & .764 & .762 \\
coupling $(\%)$ & 0.53 & 0.57 & 0.66 & 0.66 & 0.62 \\
$E_{p k} / E_{a c c}$ & 3.52 & 3.13 & 2.88 & 2.56 & 2.31 \\
$H_{p k} / E_{a c c}$ & 83.8 & 73.9 & 67.5 & 59.8 & 54.9 \\
$(\mathrm{Gs} /(\mathrm{MV} / \mathrm{m}))$ & & & & & \\
$R_{s} * Q_{0}(\mathrm{Ohm})$ & 103 & 132 & 161 & 203 & 238 \\
$R_{s h} / Q_{0}(\mathrm{Ohm} / \mathrm{m})$ & 387 & 474 & 541 & 650 & 730 \\
$Q_{0} * 10^{-10}$ & 0.83 & 1.06 & 1.29 & 1.62 & 1.90 \\
\hline
\end{tabular}

\section{SPOKE CAVITY}

As an alternative to the elliptical cavity for small $\beta$ 's a spoke cavity is under consideration (Fig. 6). This one cell cavity has been built and tested at high fields at Argonne National Lab. The advantages of such type cavity before elliptical ones are smaller dimensions and higher mechanical ridgidity. An accelerating $\pi$-mode electrical field is similar to the elliptical cavity accelerating mode but magnetic peak field on surface is defined by spoke diameter. To provide the calculations of $E_{p k} / E_{a c c}$ on the cavity surface the spokes have been made round (Fig. 7). There is an optimum of $E_{p k} / E_{a c c}$ depending on the spoke length (Fig. 8) which explains by co-dimensions of an accelerating gap and a space between the spoke and a cavity wall.

Table 2 lists some parameters for multycell spoke cavities.

An experimental stand and normal conducting 5-cell cross bar spoke cavity model have been built. First measurements show a good agreement with numerical simulations. On Fig. 10 a first result of an electric field profile measurement along a model axis is presented.

\section{ACKNOWLEDGMENTS}

The authors would like to thank Dr. Yu. Senichev for helpfull discussions, K. Sobota, A. Richert and R. Stassen for the construction of an experimental stand and the measurement help. 


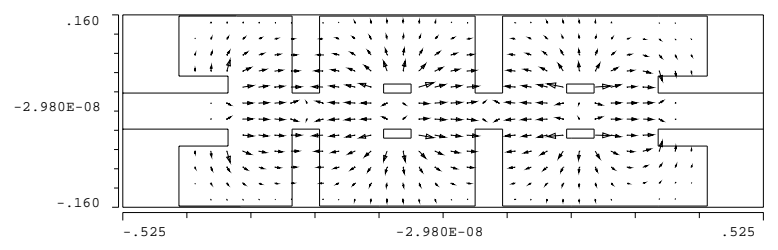

$0^{X} \underbrace{Z}$

Figure 6: Spoke Cross Bar Cavity Geometry

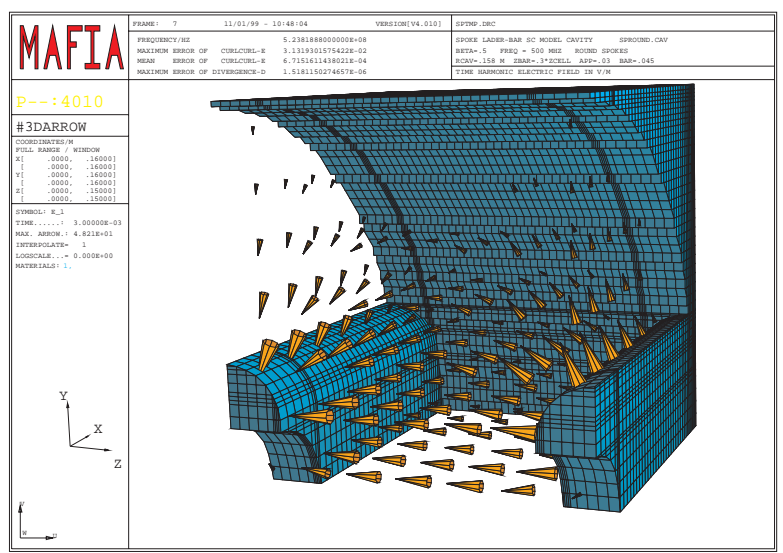

Figure 7: Electric Field in Spoke Lader Cavity (1/4 of cell is shown)

\section{REFERENCES}

[1] N. Ito et al., "Development of a Superconducting Cavity for the High Intensity Proton LINAC in JAERI", Proceedings of the XVIII Int. Linear Acc. Conf., Geneva, 1996, p.671.

[2] F. L. Krawczyk et al., "Superconducting Cavities for the APT Accelerator", Proceedings of the 1997 Particle Acc. Conf., p. 2914.

[3] D. Barni et al. "SC Beta Graded Cavity Design for a Proposed $350 \mathrm{MHz}$ LINAC for Waste Transmutation and Energy Production", EPAC'98, Stockholm, p.1870 (1998).

[4] J. R. Delayen et al., "Design and Test of a Superconducting Structure for High-Velocity Ions", LINAC'92, Ottawa, 1992.

[5] W. Braeutigam et al., this proceeding.

[6] J. H. Billen and L. M. Young, "POISSON/SUPERFISH on

Table 2: Some Parameters to Compare Spoke Cavities with Different $\beta=v / c$

\begin{tabular}{|l|c|c|c|}
\hline$\beta$ & $\mathbf{0 . 3}$ & $\mathbf{0 . 4}$ & $\mathbf{0 . 5}$ \\
\hline cell-length $(\mathrm{cm})$ & 9 & 12 & 15 \\
acc. gap $(\mathrm{cm})$ & 4.5 & 7.8 & 10 \\
transit time factor & 0.805 & 0.783 & 0.770 \\
$E_{p k} / E_{a c c}$ & 4.48 & 3.53 & 3.27 \\
$R_{s} * Q_{0}(\mathrm{Ohm})$ & 68.7 & 86.7 & 95.4 \\
\hline
\end{tabular}

